\begin{tabular}{|c|c|}
\hline 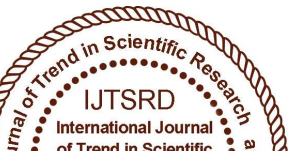 & $\begin{array}{l}\text { International Journal of Trend in Scientific } \\
\text { Research and Development (IJTSRD) }\end{array}$ \\
\hline $\begin{array}{lll} & \\
0\end{array}$ & International Open Access Journal \\
\hline and & ISSN No: $2456-6470$ | www.ijtsrd.com | Volume - 2 | Issue -3 \\
\hline
\end{tabular}

\title{
Development of a Web Content Management System in PHP
}

\author{
Vipul Bhardwaj \\ Department of Information Technology, Maharaja \\ Agrasen Institute of Technology, New Delhi, India
}

\author{
Mrs. Meenu Garg \\ Department of Information Technology, Maharaja \\ Agrasen Institute of Technology, New Delhi, India
}

\begin{abstract}
Content Management Systems are gaining importance with the spread of internet. They have considerably simplified the maintenance of digital content. Unlike other hardcoded approaches for website development, such systems do not require any technical knowledge for web publishing. They support dynamic web pages, centrally managed database systems and efficient organization of information, which makes them a dominant web development tool. This paper establishes the importance of a content management system and outlines the development of such system using technologies such as PHP and MySQL.
\end{abstract}

KEYWORDS: CMS, Web Content Management, Blog Content Management Systems

\section{INTRODUCTION}

A content management system (CMS) is a software application or set of related programs that are used to create and manage digital content. It allows publishing, editing, organizing, deleting and maintaining content from a central interface on a server.

A CMS has two main components:

\section{A. Content Management Application (CMA)}

It is a front-end application that allows user to control management of content without requiring knowledge of technical details. The application has a user interface which enables control of database and website templates.

B. Content Delivery Application (CDA)

It provides the back-end services that support management and delivery of content once it has been created in the CMA. The actual implementation is done by CDA in cooperation with CMA.

\section{A Web CMS}

A Web Content Management System (or WCMS) is the most common form of a CMS for administering web content. In fact, most of the popular content management systems are WCMS.

It is a software content management system (CMS) specifically designed for web content. It provides website authoring, collaboration and administration tools that help users with little knowledge of web programming languages or markup languages create and manage website content.

A WCMS requires the following components:

CMS Database - the central repository for all content

Template driven user interface design of CMS

An automated system to create, design and deploy the web content

A CMS management tool to manage groups, users and rights from a central control

\section{Need For A CMS}

In early days of the internet, static websites with traditional information updating methods were most commonly used. Such websites are written in plain HTML and the data is directly displayed to the user. They offer flexibility to the web developer to make every page different from the other or in adding unique features to a specific page. Upfront cost for such a website is generally low. 
However, static websites are more difficult to update than to generate. In order to implement the smallest update, one has to unnecessarily go through thousand lines of code. The user has to be technically sound to avoid introducing errors in the exercise. This becomes apparent when the user needs to update something that appears on every page, such as a Menu link, or change the website layout and design. Every page that contains the change has to be edited, and with a very large website this can be quite time consuming and costly.

Templates and content are generally tied together in a website managed without a CMS. Making a change is thus difficult without the help of a web developer. Further, such changes cannot be tracked and it is difficult to revert back to an earlier version. This gives rise to the need for an optimal solution of organising information, which a CMS fulfils.

For every business on the internet, it is necessary to capture user's attention with interactive content. Further, every website needs to implement security features to guard itself against potential attacks. Plain HTML pages are insufficient in achieving these objectives and an organized content management is required.

As information is valuable on the internet, there is a greater need for content integration between website and various other channels. Such large websites require a central administration for web publishing and access to multiple authors spread across the globe. While single page websites may work without a CMS, most multi-page websites on the internet need coherence in website design, structure and content organization. In order to account for these requirements, the presence of a CMS becomes crucial.

\section{Tools To Implement Blog CMS}

The development project makes use of the following languages/tools:

\section{HTML}

It is used to define basic web page structure including titles, forms and the static content.

\section{CSS}

It is used to control display settings such as margins, padding, and text size to ensure user friendly output.

\section{Javascript}

It is a client-side scripting language which is directly embedded into HTML. The project makes use of Javascript to control properties of various HTML elements and validating form data.

\section{PHP}

It is an open source server-side scripting language. The project makes an extensive use of PHP to create dynamic web pages which collect content from HTML forms into the database and display content fetched from the database to the users.

\section{MySQL}

MySQL is an open source database server application. It is invoked through queries embedded within the PHP scripts.

\section{Bootstrap}

Using bootstrap CSS framework, responsive and mobile friendly design is implemented with predefined classes. Bootstrap grids, buttons, panels, navbars are directly used in the project.

\section{B BlogGMS}
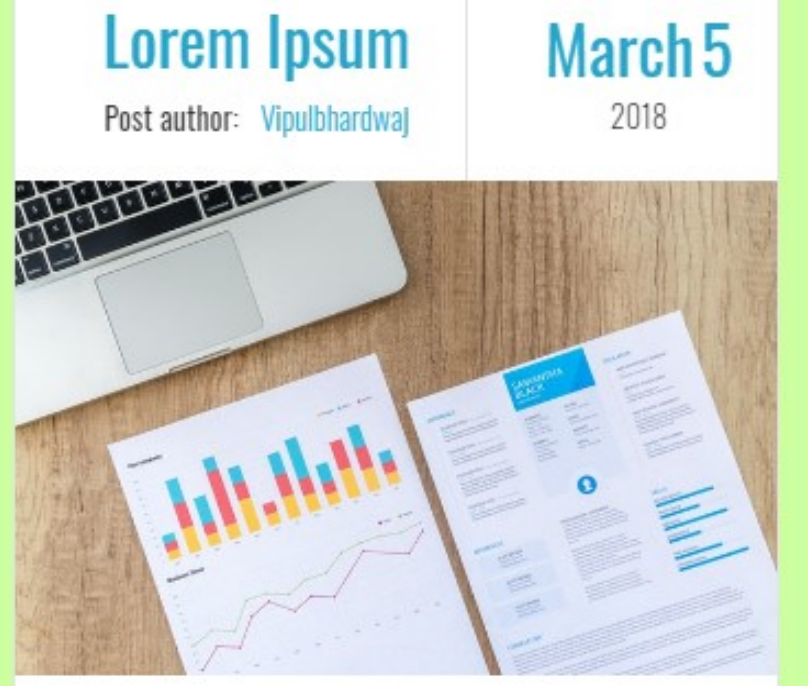

Lorem ipsum dolor sit amet, consectetur adipiscing elit. Nullam tincidunt, nunc non egestas lacinia, enim diam laoreet tellus, vitae blandit tellus mi eu nisi. Sed sit amet diam lacinia, tristique augue placerat, sodales quam. Suspendisse nec blan.

D. Html I Comment Read More

Figure: A snapshot of Blog CMS 


\section{Implementation Details}

\section{Database}

The database for the Blog CMS is implemented using following tables:

\section{Posts}

The table stores all posts referenced by the primary key post id and has fields date, title, author, image, category, data and views count. Any new post added is stored here and is retrieved when a user requests for the page.

\section{Users}

All relevant details of users such as id, first name, last name, username, password, email and role are stored in this table.

\section{Media}

The table includes fields for media id and the path to its location on server.

\section{Comments}

All comments submitted to any post are stored with fields including name, date, post id, comment id, email, content and status are stored here.

\section{Categories}

This table lists the categories defined by the author along with it their ids and names.

\section{Features implemented}

1. Default templates

The CMS generates standard output template built using HTML and CSS that can be automatically applied to new and existing content, allowing the appearance of all content to be changed from one central place. This separates the content from the presentation.

\section{Easily editable content}

Content is separated from the visual presentation of a site, which makes it much easier and quicker to edit and manage. WYSIWYG (What You See Is What You Get) editing tool is used to enable users edit content without need to know technical details.

\section{Access control}

Session management and user roles are clearly defined which restricts unauthorized users from making any modifications. Further, registered users have limited access privileges.
4. Integrated Analytics

Every page request triggers an updation of the view count which is reported on per-post basis to logged in users.

\section{Security}

Through inbuilt crypt function in PHP, the data and passwords are encrypted to make them secure.

\section{Workflow management}

Workflow management is implemented to assist teams with the process of managing and publishing content. The workflow used is a publishing queue where a post awaits final approval from the administrator before it appears on the website.

\section{Future Scope}

There are around 1.8 billion websites on the internet and it is estimated that over half of them use a CMS. Wordpress, an open source CMS, alone powers nearly $30 \%$ of the websites on Internet. Extending capabilities of existing CMS using multilingualism, content distribution systems and social media integration can open many new possibilities.

With rapidly advancing technology and accessibility of internet, Content Management Systems are adding new capabilities using Artificial Intelligence and cloud based technologies. Many WCMS have started offering cognitive and analytical capabilities to assess how content has come across to readers, which tone resonates with which audience, and so forth. A new model of content management as Experience-as-aService (EaaS) has gained momentum. Universal web accessibility and web security are new concerns of the emerging content management systems.

\section{REFERENCES}

1) http://searchcontentmanagement.techtarget.com/d efinition/content-management-system-CMS

2) https://en.wikipedia.org/wiki/Web_content_manag ement_system

3) http://www.spiderwriting.co.uk/staticdynamic.php

4) https://www.amityweb.co.uk/blog/static-ordynamic-content-managed-website-which-one-isbest

5) https://websitesetup.org/popular-cms/

6) http://www.cms-connected.com/NewsArchive/December-2017/5-Web-ContentManagement-Trends-for-2018 